\title{
Protótipo de Bengala Inteligente de Baixo Custo para o Auxílio de Deficientes Visuais
}

\author{
Alice Freitas, Atos Lima, Marcelo Santos \\ Instituto Federal do Sertão de Pernambuco (IF Sertão-PE) \\ Departamento de Informática - Campus Salgueiro \\ BR 232, Km 508 - 56000-000 - Salgueiro - PE - Brasil \\ \{alice.freitas, marcelo.santos\} @ifsertao-pe.edu.br, \\ atosss.lehotmail.com
}

\begin{abstract}
Based on the need to support people with visual impairment, the goal of this article seeks to present a prototype created with the purpose of increasing the accessibility and security for this type of user. The prototype consists of a handmade cane that has attached itself an ultrasonic sensor which alerts the user of possible obstacles in your path through a sound system. This sound signal varies according to the degree of proximity of an obstacle by increasing or decreasing the frequency of this signal. Besides that, the prototype aims an integration with a smartphone in order to use sensors from the smartphone through a Bluetooth connection.

Resumo. Baseando-se em uma necessidade de amparo a deficientes visuais, o objetivo desse artigo é apresentar um protótipo criado com a finalidade de aumentar a acessibilidade e segurança para esse tipo de usuário. $O$ protótipo é constituído de uma bengala artesanal que tem acoplada a si um sensor ultrassônico o qual alerta o usuário de possíveis obstáculos em seu trajeto através de um sistema sonoro. Tal sinalização sonora varia de acordo com o grau de proximidade do obstáculo, aumentando ou diminuindo a frequência desse sinal. O protótipo visa ainda a interação com um smartphone com o objetivo de utilizar os sensores do próprio smartphone através de uma comunicação via Bluetooth.
\end{abstract}

\section{Introdução}

Segundo o censo realizado pelo IBGE em $2010^{1}$, a deficiência visual é o tipo de deficiência mais comum no Brasil atingindo cerca de 3,6\% dos brasileiros. Nesse contexto, a inclusão social torna-se importante e vem crescendo devido o surgimento de diversas tecnologias e projetos que auxiliam nesse processo. Nos últimos 12 anos, por exemplo, o número de deficientes visuais no ensino básico cresceu $381 \%$. Já no ensino superior esse número cresceu $475 \%$, passando de 5.078 estudantes para $29.221^{2}$.

Nesse cenário, a acessibilidade conceituada pela Lei 10.098 como sendo a possibilidade e condição de alcance para a utilização com segurança e autonomia dos espaços, mobiliários e equipamentos urbanos das edificações, transportes e dos

\footnotetext{
${ }^{1}$ biblioteca.ibge.gov.br/visualizacao/periodicos/94/cd_2010_religiao_deficiencia.pdf

${ }^{2}$ http://www.brasil.gov.br/educacao/2015/09/numero-de-pessoas-com-deficiencia-nas-escolascresce-381-em-12-anos
} 
sistemas e meios de comunicação por pessoa portadora de deficiência ou com mobilidade reduzida, refere-se a dois aspectos que estão sujeitos a problemas semelhantes no que diz respeito à existência de barreiras que são interpostas às pessoas com necessidades especiais: o espaço físico e o espaço digital. (TAVARES FILHO et al., 2002). Baseando-se nesse conceito de acessibilidade surge uma necessidade de atender de diversas formas o público a qual essas condições fazem parte da rotina diária.

Portanto, considerando os cerca de 7 milhões de deficientes visuais no Brasil, temos como objetivo criar uma bengala inteligente que propicie o aumento da segurança no translado e consequentemente a melhora na qualidade da locomoção de pessoas com algum tipo de deficiência visual. Cabe ressaltar que apesar de existirem atualmente diversas tecnologias que auxiliam os cegos na leitura, aprendizado, comunicação, suporte para visão artificial e até mesmo transplantes modernos de córneas ${ }^{3}$. Infelizmente muitas das opções disponíveis não são de fácil manuseio ou não possuem baixo custo para os deficientes visuais.

Dentro desse projeto, Internet das Coisas (IoT) (GUBBI et al., 2013; WHITMORE; AGARWAL; DA XU, 2015) surge como um paradigma que permite a conexão e o processamento dos dados coletados pela bengala em um smartphone com acesso à Internet através de uma comunicação Bluetooth. Essa capacidade de comunicação com um outro dispositivo abre um leque de possibilidades interativas e facilidades no processamento dos dados. Dessa forma, nessa etapa do projeto faremos uso do sensor de vibração do próprio smartphone para alertar o usuário sobre algum obstáculo. Dessa forma, o objetivo deste artigo é desenvolver uma bengala eletrônica inteligente, constituída de material reciclado ou artesanal, de baixo custo, com suporte tecnológico de sensores e dispositivos sonoros que atuem de forma a interagir com o usuário deficiente visual em sua locomoção, evitando obstáculos e priorizando a segurança do translado do usuário usando também o sensor vibratório do próprio celular do usuário.

A utilização da bengala para constituir o protótipo partiu da necessidade vista dentro do próprio campus do Instituto Federal do Sertão de Pernambuco (IF Sertão$\mathrm{PE})$ que hoje atende a uma grande quantidade de alunos com diversos tipos de deficiência, beneficiando-os dos resultados deste protótipo e estimulando a busca dentro da pesquisa e inovação tecnológica. $O$ ponto de partida para montagem do protótipo é o acoplamento de sensores em uma bengala para identificação de obstáculos os quais normalmente a extremidade de uma bengala usada por deficientes não alcançaria. Os sensores são conectados a um arduino, dispositivo para automação eletrônica, e estes atuarão de forma a realizar a verificação de obstáculos durante o trajeto que o deficiente visual se movimentará, interagindo assim com o arduino para que este possa emitir o alerta em forma sonora para o usuário através de um buzzer. Todo o processo de testes no circuito da automação foi elaborado com auxílio de um deficiente visual na busca pela precisão da resposta ao usuário e assegurando a sua segurança.

\footnotetext{
${ }^{3}$ http://www.saudebemestar.pt/pt/clinica/oftalmologia/transplante-de-cornea/
} 
O artigo está organizado da maneira a seguir: seção 2 apresenta os trabalhos relacionados; seção 3 o desenvolvimento do protótipo, seção 4 considerações finais e trabalhos futuros e na seção 5 as referências.

\section{Trabalhos Relacionados}

A busca por tecnologias que facilitem a realização de atividades executadas por deficientes físicos, sobretudo deficientes visuais não é novidade e sim recorrente fonte de diversas pesquisas e novos projetos na área. Dentro do que concerne a locomoção, existem variados trabalhos que consistem em assegurar a melhor execução do trajeto dos deficientes visuais com uma maior segurança.

O projeto Argos (FILHO et al, 2010) é baseado no desenvolvimento de um dispositivo de auxílio à locomoção de deficientes visuais a partir de uma pulseira microcontrolada criada por estudantes da Centro Universitário do Pará (CESUPA). O dispositivo desenvolvido é preso ao pulso do usuário e funciona criando um campo ultrassônico que permite que o dispositivo colete informações dos obstáculos no caminho do usuário. Ao processar os sinais coletados é tomada a decisão sobre a emissão de um sinal vibratório de alerta (|Wilson et al., 2015) referente a um obstáculo próximo. O custo orçado em 2011 para desenvolvimento do projeto foi de cerca de $\mathrm{R} \$ 163,75$ (FILHO et al, 2011).

Pesquisadores da USP (Universidade de São Paulo) também trabalham nesse tipo de tecnologia de varredura do ambiente por meio de sensores para interagir com o deficiente visual em sua locomoção. O projeto SoundSee (AGÊNCIA USP, 2016) desenvolvido no Instituto de Ciências Matemáticas e de Computação (ICMC) em São Carlos projetou um dispositivo que emite sons no ambiente para poder calcular a posição de obstáculos próximos, similarmente ao trabalho desenvolvido por FILHO et al. Ambos os trabalhos relacionados tratam da problemática de forma bem objetiva, com leitura sensorial dos obstáculos no trajeto e o uso de microcontrolador para executar a função de processamento de dados e alerta ao usuário, seja ele por forma sonora ou vibratória.

Saaid et al. propõe um projeto de uma bengala dotada de um leitor de RFID (Radio Frequency Identification). Os autores nomearam o projeto de RFIWS (Saaid et al.). A ideia é colocar tags RFID em calçadas para guiar o deficiente visual por um caminho considerado seguro. O projeto baseou-se na utilização de frequências UHF (Ultra High Frequency) que permitem um range de detecção de 1 a 3 metros.

De forma similar, podemos encontrar na literatura outras aplicações que auxiliam deficientes visuais através do microcontrolador Arduino. O projeto Tacit, desenvolvido pela Grathio Labs criou uma luva que funciona como um sonar que possibilita a locomoção de deficientes visuais em ambientes desconhecidos. Seu funcionamento é relativamente simples, os sensores montados na luva medem a distância dos objetos e traduzem esta informação na forma de pressão no pulso do usuário. A capacidade de avaliação vai de uma polegada a dez pés de distância (equivalente a 2,5 centímetros até 3 metros) e a resposta é de apenas alguns décimos de segundo (FUTURE LAB, 2016).

Nesse contexto, um outro exemplo é o boné detector de obstáculos (BONÉ INTELIGENTE, 2016) para deficientes visuais foi construído com auxílio do Arduino por uma equipe de universitário da UFSC, Campus Blumenau. O boné 
funciona em três modos de sinalização para o portador, sendo eles vibratórios (emite uma leve vibração na cabeça, através do vibracall), sonoro (emitindo um som através do buzzer), ou ambos sinais simultaneamente. (FURINI, 2016)

Os trabalhos citados expõem o que foi encontrado nessa linha de automação com uso de microcontroladores para deficientes visuais. Os quais forneceram embasamento teórico para o protótipo desenvolvido neste trabalho. No nosso trabalho em específico também foi utilizado o microcontrolador arduino (MCROBERTS, 2011) por se tratar, atualmente, de um dispositivo de automação de baixo custo e com grande material técnico disponível que auxiliam na replicação do projeto. Assim, temos uma maior facilidade de alterações futuras no protótipo de forma a sofisticá-lo e atender demandas mais específicas. O custo total do projeto é orçado em R\$ 100,00, considerando o módulo arduino, o sensor ultrassônico, o módulo Bluetooth, um buzzer, e o restante dos materiais sendo de uso reciclável, obtendo-se assim um custo relativamente baixo. Outro ponto que merece destaque é a conectividade com o smartphone que permite a utilização de sensores do próprio aparelho do usuário, diminuindo o custo total do projeto ao alertar o usuário sobre obstáculos através do celular. Além disso, o uso do arduino com o módulo Bluetooth conectado a um smartphone permite extensões do projeto como, por exemplo, o envio de informações de localização do usuário para um acompanhante ou familiar através da Internet durante a utilização da bengala inteligente.

\section{Desenvolvimento do Protótipo}

O desenvolvimento do protótipo ocorreu dentro do campus do IF-Sertão de Pernambuco devido a necessidades detectadas por estudantes da rede de ensino do próprio instituto. Nesse contexto, visando a atender ao requisito de baixo custo para produção da bengala inteligente, a sua construção pode ser constituída por canos de plástico, um material reciclável e de baixo custo, que conta com um design bem simples, montado de forma similar a uma bengala tradicional em comprimento e punho para utilização, e sua constituição não afeta sua funcionalidade.

Afim de atender aos objetivos pretendidos no projeto, temos acoplado junto a bengala artesanal o dispositivo para automação eletrônica conhecido como Arduino (Figura 1). O modelo utilizado é um dos mais recente, conhecido como Arduino Uno (MCROBERTS, 2011) que está dentre as placas programáveis que possui um dos mais baixos custos.

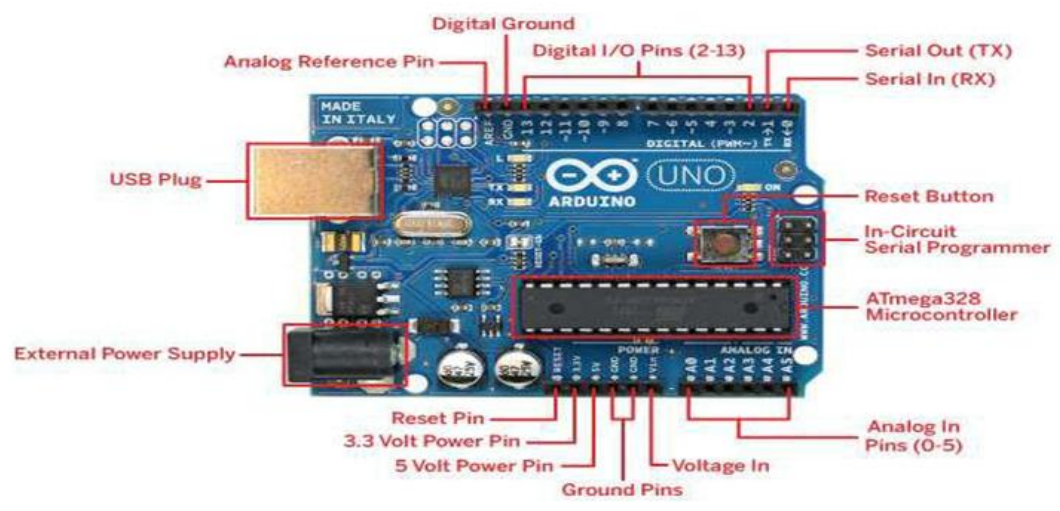

Figura 1. Arduino Uno 


\subsection{Estrutura Física do Protótipo e seus Componentes}

Um dos principais componentes para realização do projeto é o sensor ultrassónico HC-SR04 (Figura 2) que possui uma ótima precisão regulável entre $2 \mathrm{~cm}$ e $4 \mathrm{~m}$. Além disso, possui baixo custo no mercado. Este módulo possui um circuito pronto com emissor e receptor acoplados e 4 pinos (VCC, Trigger, ECHO, GND) para medição.

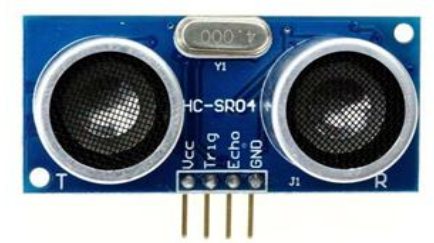

Fig ura 2. Sens or Ultras s ónico HC-S R04

O funcionamento do sensor HC-SR04 se dá através da emissão e captura de um sinal sônico. Após o lançamento do sinal através da primitiva ECHO, o sensor acoplado ao Arduino interpreta os obstáculos do ambiente através do retorno do sinal. No protótipo se esse retorno exceder a uma distância menor do que 2 metros, um sinal é emitido através de um buzzer, onde o mesmo irá variar a intensidade do som emitido de acordo com a proximidade do objeto captado. Assim, busca-se evitar o uso abusivo do sinal sonoro e o mesmo desligará o som quando não existir obstáculos maiores do que 2 metros.

A distância de detecção de obstáculos foi alcançada através de testes em locais fechados e abertos onde foram efetuadas simulações com a bengala e os possíveis obstáculos para o deficiente, sendo necessário encontrar um coeficiente seguro da distância entre o obstáculo e o deficiente, este caminhando em uma determinada velocidade média, para detecção e sinalização.

Dessa forma, utilizando a estrutura física de canos de plásticos conectados um a outro o sensor deve ser posicionado $90^{\circ}$ em relação ao chão para mais precisa possível verificação do obstáculo em relação ao usuário da bengala, as conexões dos canos facilitarão a disposição dos condutores, a alimentação de $6 \mathrm{~V}$ contínua e com uma caixa de passagem simples utilizada em instalações elétricas haverá o acoplamento com o Arduino exibido na Figura 3a. O buzzer será conectado ao Arduino e a estrutura da caixa de passagem, sendo posicionado por traz da caixa facilitando a ligação do seus condutores e sua fixação, assim como também a emissão sonora para o usuário, exibido na Figura $3 b$.

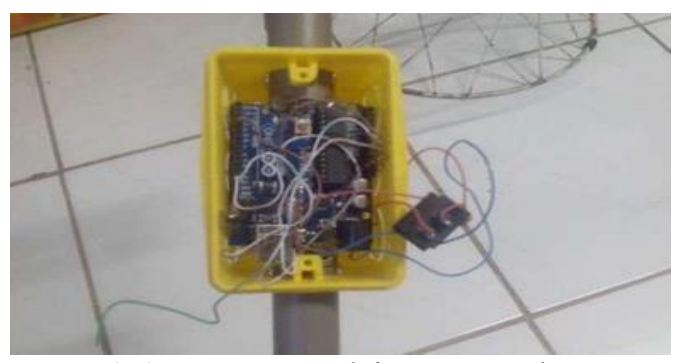

(a )Es trutura Fís ica Bengala com Arduino

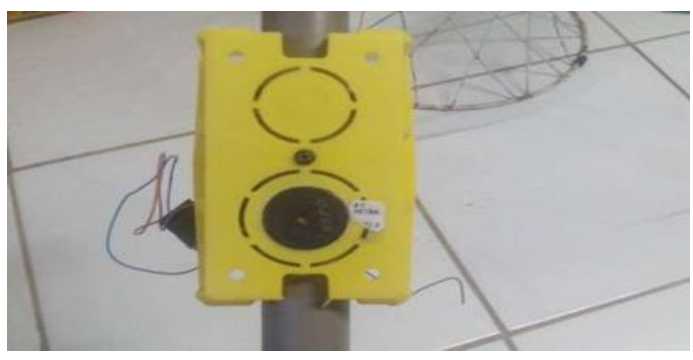

(b) Es trutura Fís ica Bengala com Arduino e Buzzer

Figura 3. Es trutura do Arduino 
$\mathrm{Na}$ finalização da estrutura, vista na Figura 4, a colocação do sensor ultrassónico HC-SR04 fica numa altura mediana em relação a extremidade que irá tocar o chão da bengala, e com perfuração e colagem de fácil manutenção. Nota-se a facilidade da montagem sem comprometer o uso do protótipo e também com boas possibilidades de adequação em termos de dimensão, manutenção e/ou possíveis e futuras melhorias.

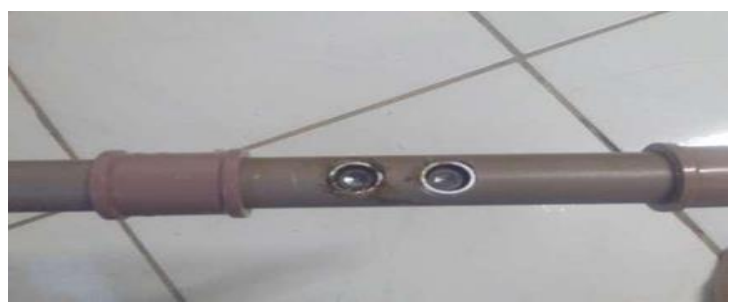

Figura 4. Es trutura Fís ica Bengala com Sens or

A alimentação para alimentar o Arduino Uno consiste de $6 \mathrm{~V}$ de corrente contínua, neste caso foi utilizado 4 (quatro) pilhas de 1,5V que devem ser colocadas na divisão central do cano para que não haja nenhuma força ou peso adicional que venha a influenciar no funcionamento do sensor. No entanto, o uso de uma bateria $9 \mathrm{~V}$ será opcional de acordo com o tipo de reprodutor sonoro que possa ser utilizado em alguma outra aplicação específica ou melhoria do protótipo. Quando fixado apenas o buzzer ao sistema, o mesmo por possuir baixa potência e uma necessidade pequena de consumo de corrente elétrica não necessita de bateria, porém para fones de ouvido sem fio, ou alto-falantes de maior potência, bem como outros dispositivos, o uso da bateria de $9 \mathrm{~V}$ deverá ser avaliado.

\subsection{Código do Protótipo}

De acordo com o modo de programação do Arduino, a implementação do código ocorreu através da sua própria IDE ${ }^{4}$. $\mathrm{O}$ início da implementação pode ser visto na Figura 5. Declara-se então as variáveis para acionamento, controle do sensor ultrassónico e buzzer (linhas 1 a 6 ).

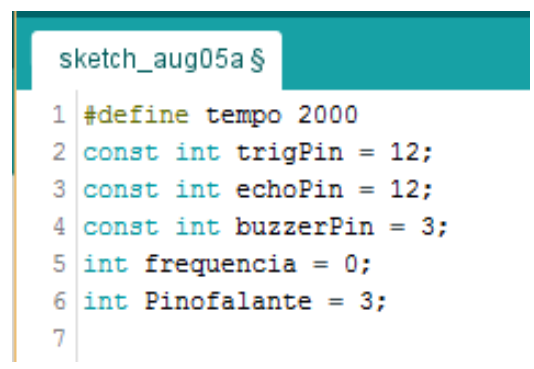

Figura 5. Variáveis declaradas

Observa-se na primeira linha o uso do "\#define", que define uma variável chamada "tempo" com o valor de 2000 milissegundos. Em seguida, na segunda linha o termo "const" servirá para que o valor atribuído as variáveis trigPin, echoPin e buzzerPin não sejam alterados, já que os mesmos são pinos digitais sensíveis que ligam o sensor e buzzer ao Arduino para que os sinais coletados possam ser processados.

\footnotetext{
${ }^{4}$ https://www.arduino.cc/en/Main/Software
} 
Seguindo com o código exposto na Figura 5, na Figura 6 temos a função $\operatorname{Setup}()$ informando quais pinos serão entradas ou saídas no circuito (Inputs/Outputs).

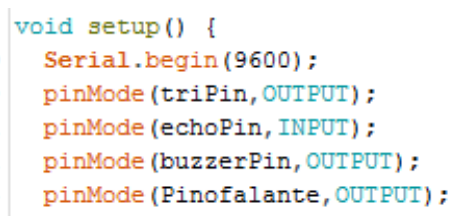

Figura 6. Definição dos pinos de entrada e s aída (Inputs/Outputs)

O comando Serial.begin() servirá para efetuar a comunicação serial auxiliando no envio e recebimento de dados da IDE. Em seguida o comando pinMode() servirá para indicar se o pino digital será de saída ou entrada. Assim, a linha 10, "pinMode(trigPin, OUTPUT);" indicará que a variável criada anteriormente será uma pino de saída no nosso circuito. Já na linha 11, "pinMode(echoPin, INPUT);" define-se um pino de entrada e segue-se a mesma lógica para os demais pinos.

Dando seguimento, é exposto na Figura 7 o início da função loop() onde está toda a parte operacional do código.

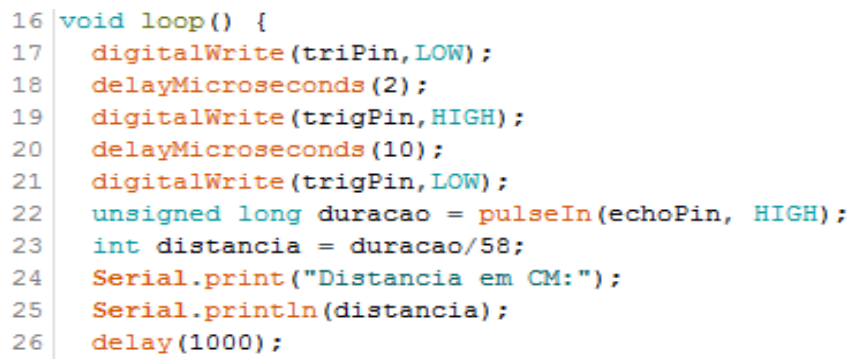

Figura 7. Função loop()

Cuidados em particulares devem ser tomados nas linhas 16 a 43, dado que a esse trecho do código pode comprometer a integridade dos componentes do circuito. Nas linhas 17 e19, a função digitalWrite() tem como principal finalidade configurar o estado da porta como alto( $\mathrm{HIGH}$ ) ou baixo (LOW), definindo qual será a voltagem recebida e qual a resistência em ohms destas portas. A função delayMicroseconds() nas linhas 18 e 20 indicarão quantos microssegundos a porta permanecerá nesse estado.

$\mathrm{Na}$ linha 22, é declarado uma variável do tipo long que não pode assumir valores negativos devido ao uso da palavra reservada unsigned. Em seguida a função pulseIn() servirá para receber a informação obtida pelo echoPin. No nosso programa, no momento em que o echoPin recebeu o estado de HIGH, "pulseIN(echoPin, HIGH);", o Arduino iniciará o echoPin como HIGH e começará uma cronometragem até que o echoPin se torne LOW. O sinal LOW surgirá quando um obstáculo surgir próximo ao sensor, retornando o valor para a variável "duracao". A linha 23, "int distancia = duracao/58", tem como objetivo transformar o dado obtido em centímetros.

As funções Serial.println() e delay(), servirão apenas como teste para a verificação do correto funcionamento do sensor, já que os mesmos servirão para 
mostrar em uma tela o valor que foi atribuído e com que frequência será mostrada a variável distancia .

A Figura 8 exibe a configuração das condições quando o sensor irá alertar ou não o usuário, sendo uma continuação da função $\operatorname{loop}()$ exibida anteriormente.

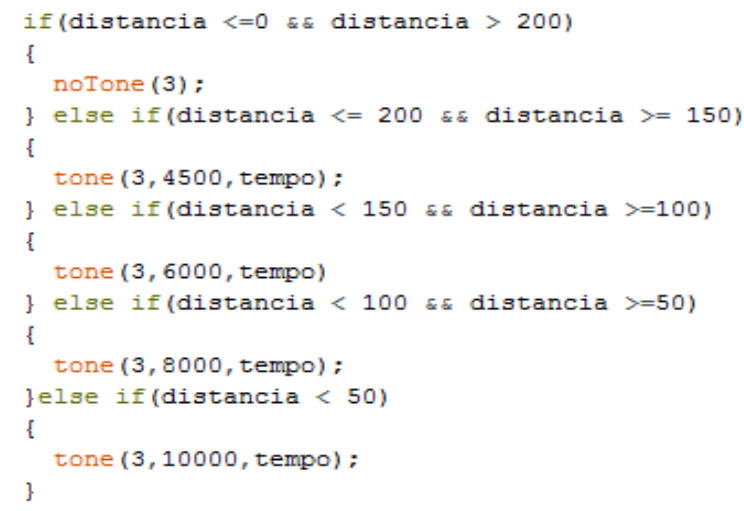

Figura 8. Continuação da função loop ()

Foram usadas estruturas de condições simples baseadas em If's e Elses, que irão se basear na variável distancia recebida pelo echoPin e comparadas com as distâncias desejadas para seu acionamento em centímetros. A função tone() irá atribuir um Tom, frequência e tempo ao pino 3, declarado no início com o PinoFalante, sendo o emissor sonoro, logo percebe-se que a frequência irá alterar de acordo com a proximidade do objeto obtido, sendo assim aumentando a intensidade do sinal porém mantendo o tempo desse Tom, já atribuído a variável tempo.

Após a montagem e testes no protótipo foram observados problemas em tempo de execução no dispositivo acoplado a bengala, este agiu com que o sensor aumentasse o tempo de início do Tom (sinal de frequência e onda quadrada para alimentar o buzzer), trazendo um péssimo funcionamento para a bengala já que se houver um obstáculo repentino o bip do buzzer não irá funcionar no tempo necessário por possuir um delay significante. Em outros momentos, o buzzer emitia sons mesmo sem obstáculos, pois eram gerados BUG's onde as condições não eram finalizadas no programa após detectarem os objetos, logo o uso do noTone() para esse código simplificado foi indispensável para precisão do funcionamento pois interrompe a geração de sinal.

\subsection{Conectividade com smartphone}

Como continuação do projeto, ainda em fase de teste, há o desenvolvimento da interação entre a bengala inteligente e o smartphone através de um módulo bluetooth conectado ao Arduino. Esse nível de conectividade abre diversas frentes a serem exploradas. A primeira delas é simplesmente a utilização do sistema de vibração do próprio celular para alertar o usuário sobre algum obstáculo detectado pela bengala. Assim, na Figura 9 podemos observar a adição do módulo Bluetooth RS232 HC-05 que permite o envio de informações para o celular.

O aplicativo que executará no Android e se comunicará com o Arduino através da conexão Bluetooth pode ser desenvolvido através da ferramenta AppInventor do MIT, disponível no endereço http://appinventor.mit.edu/. Essa ferramenta é gratuita e oferece suporte para o desenvolvimento de aplicações que 
V Congresso Brasileiro de Informática na Educação (CBIE 2016)

Anais dos Workshops do V Congresso Brasileiro de Informática na Educação (CBIE 2016)

podem interagir, por exemplo, com todos os sensores do celular (Figura 10). Outras opções são o uso dos aplicativos Bluetooth $\mathrm{SPP}^{5}$ e microController $\mathrm{BT}^{6}$.

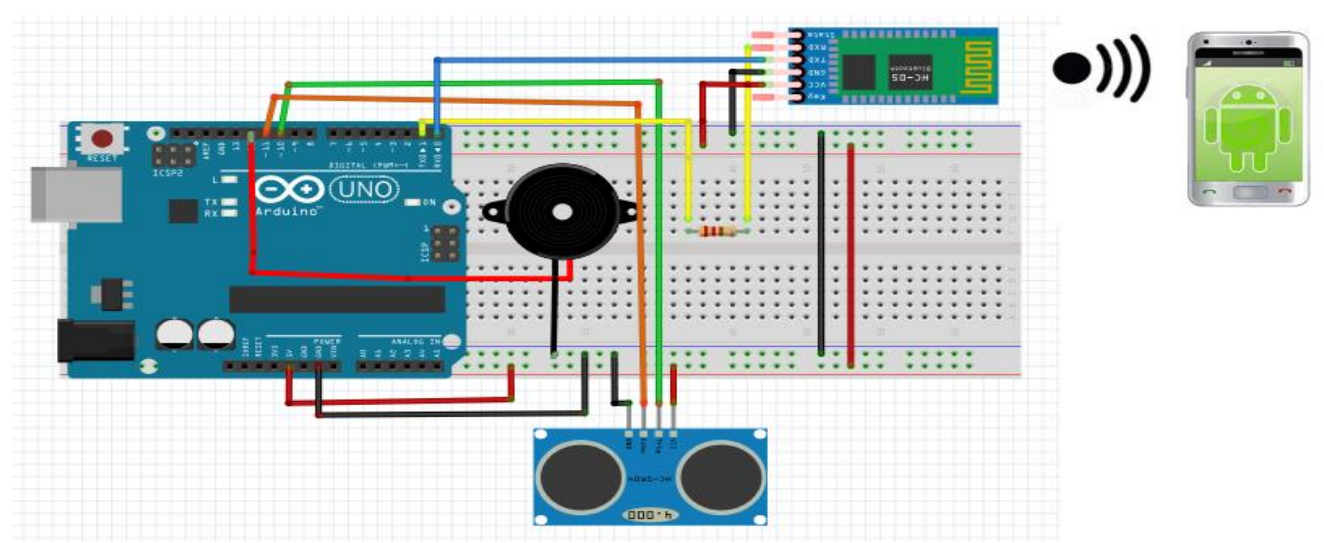

Fig ura 9. Circuito elétrico completo do proje to

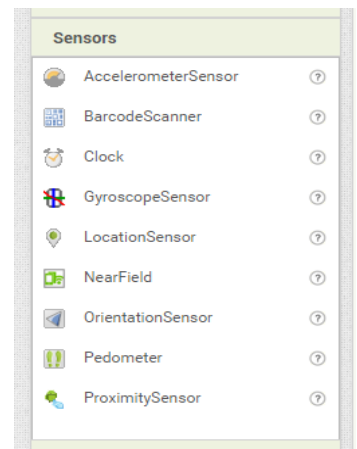

Figura 10. Menu do AppInventor

Toda a parte relacionada a execução dos comandos de comunicação do módulo Bluetooth com o Smartphone através do Android, é feita primeiramente através da configuração do código e sinalização das informações para a portas seriais do módulo, TX, RX, que se comunicam após ativação do bluetooth no smartphone e pareamento dos mesmos, sendo estes recebidos e codificados em seus objetivos finais de sinalização ao usuário através do AppInventor.

Dessa forma, atualmente o projeto encontra-se na fase de finalização do aplicativo para o Android que veio após os testes iniciais com a bengala e sensoriamento dos obstáculos ao redor do usuário com sucesso.

\section{Considerações Finais e Trabalhos Futuros}

Este trabalho propôs o protótipo de uma bengala inteligente que auxilia deficientes visuais a se locomoverem com melhor qualidade e segurança através da emissão de alertas sonoros e vibratórios diante da detecção de obstáculos.

O projeto desenvolvido tem como um de seus objetivos alcançar um custo baixo e para isso foram utilizados como principais componentes o microcontraldor

\footnotetext{
${ }^{5} \mathrm{https}$ ///play.google.com/store/apps/details?id=mobi.dzs.android.BLE_SPP_PRO

${ }^{6} \mathrm{http}: / /$ microcontroller-bt.android.informer.com/
} 
Arduino, um buzzer, um módulo Bluetooth e o próprio celular do usuário. O Arduino pode se conectar através de uma conexão Bluetooth a diversos dispositivos como notebooks, tablets e celulares, o que permite uma maior variedade de alertas que podem ser emitidos. Pode-se, por exemplo, emitir um sinal vibratório para um dispositivo externo junto ao corpo do usuário como o seu próprio celular. Assim, após a finalização inicial do protótipo da bengala inteligente, os testes de detecção dos obstáculos foram realizados afim de obter uma melhor calibração na sensibilidade para detecção de obstáculos, apresentando resultados satisfatórios. Como resultado final, todo código fonte do projeto será disponibilizado gratuitamente após as realizações de testes do aplicativo Android, ainda em desenvolvimento. Com o código estável disponível, qualquer um poderá melhorar e expandir o escopo do projeto.

Desta forma, a pesquisa e criação deste protótipo é apenas de um ponto de partida para novos e mais complexos protótipos como, por exemplo, quando adicionamos um sinalizador motorizado, reconhecimento de voz e monitoramento geográfico, atendendo cada vez mais de forma precisa a anseios da comunidade de deficientes visuais no que concerne a sua locomoção. Há também a possibilidade de conexão do arduino com redes wifi com a aquisição do módulo wireless ESP8266, podendo trabalhar como um Ponto de Acesso (Acess Point) ou como uma Estação (Station), enviando e recebendo dados. Dessa forma, como trabalhos futuros vislumbramos o mapeando de ambientes, enviando informações para uma leitura detalhada do trajeto para o usuário ou um acompanhante ou familiar que não esteja no local e deseje se assegurar da segurança do deficiente visual.

\section{Referências}

FUTURE LAB - http://futurelab.com.br/site/futureblog/projeto-com-arduino-ajudaos-cegos-a-se-locomoverem/. Acessado em 10/09/2016

BONÉ INTELIGENTE - Vídeo explicativo: disponível em: http://1080plussimulcastingvideochatplayer.website/Projeto_Ardu\%C3\%ADnoBon \%C3\%A9_para_deficientes_Visuais/CeC9hpdJhd8.video. Acessado em 10/09/2016

FURINI Blog - Disponível em: http://furini.blog.br/?p=234. Acessado em 10/09/2016

MCROBERTS, Michael. Arduino Básico. São Paulo: Novatec, 2011.

TAVARES FILHO, J. P., MAZZONI, A. A. RODRIGUEZ, A .M. e ALVES, J. B. M. (2002) "Aspectos ergonômicos da interação com caixas automáticos bancários de usuários com necessidades especiais características de idosos". In: Congresso Iberolatinoamericano de Informática Educativa Especial, 3. Anais em CD, Fortaleza - Brasil, 2002.

GUBBI, J. et al. Internet of Things (IoT): A vision, architectural elements, and future directions. Future Generation Computer Systems, v. 29, n. 7, p. 1645-1660, 2013.

WHITMORE, A.; AGARWAL, A.; DA XU, L. The Internet of Things-A survey of topics and trends. Information Systems Frontiers, v. 17, n. 2, p. 261-274, 12 abr. 2015. 